\title{
Configuration Management for Wendelstein 7-X
}

R. Brakel ${ }^{*}$, P. v. Eeten, D. A. Hartmann, K. Henkelmann, J. Knauer, K. Müller, A. OkkengaWolf, U. Wenzel

Max-Planck-Institut für Plasmaphysik, EURATOM-Association, Wendelsteinstr. 1, 17491 Greifswald, Germany

* corresponding author, e-mail: brakel@ipp.mpg.de, phone: 00493834882300

\begin{abstract}
A complex system like the large superconducting Wendelstein 7-X stellarator necessitates a dedicated organizational structure which assures permanent consistency between the requirements of its system specification and the performance attributes of all its components throughout its life time. This includes well defined processes and centrally coordinated information structures. For this purposes the department Configuration Management (CM) has recently been established at W7-X. The detailed tasks of CM for W7-X are oriented along common CM standards and comprise configuration identification, change management, configuration status accounting and configuration verification. While the assembly of W7-X is proceeding some components are still under procurement or even under design. Thus design changes and non-conformances may have a direct impact on the assembly process. Highest priority has therefore been assigned to efficient control of change and nonconformance processes which might delay the assembly schedule.
\end{abstract}

Keywords: Configuration Management

\section{Introduction}

The complexity of a large fusion device like the superconducting Wendelstein 7-X stellarator [1] necessitates a dedicated organizational structure which assures permanent consistency between the requirements of its system specification and the performance attributes of all its components throughout the life time of the project. For this purpose the department Configuration Management (CM) has been established at W7-X in 2007, being part of the 
division Design and Configuration, which also hosts the departments Design Office and Configuration Space Control. The structural reorganization concentrates the responsibility for CM tasks, which was previously distributed among various departments, in one department. The implementation of this organizational unit was essentially driven by the circumstance, that while the assembly of W7-X is proceeding some of its components are still under procurement or even under design. Thus design changes and non-conformances may have a direct and significant impact on the assembly process and fast handling of change and nonconformance processes which might affect the assembly schedule has therefore been assigned the highest priority for CM. Additionally, the proximity of $\mathrm{CM}$ to the central Design Office, being responsible for the generation of design models and fabrication drawings, and to the department Configuration Space Control, which controls the geometrical configuration space both within and outside the W7-X cryostat, supports fast assessment of and reactions on configuration problems.

The specific tasks of CM for W7-X are oriented along common CM standards as ISO 10007:2003 [2]. They comprise configuration identification, change management, configuration status accounting and configuration verification. CM provides access to the complete configuration information via a Product Lifecycle Management (PLM) system based navigation tool and takes care that starting from a central master document for each component the user is guided to the information of interest, e.g. the position of the component in the project structure and CAD assembly, the specifications, the history of design changes and variances and the interface descriptions. CM controls all change processes, preserves configuration control at the interfaces, ensures documentation and consistent implementation of all changes and variances, and provides orderly communication of change information within the project. The status of the CM processes is documented and controlled via a data base.

In addition to the close interaction with the departments Design Office and Configuration Control, particularly close logical interfaces of CM exist with the departments Quality Management (with respect to procedures, quality standards, verification, auditing), Documentation (project-structure, PLM system), Project Control (cost and schedules), and the division Engineering (calculations in support of design, design reviews). 


\section{Configuration identification}

\subsection{Project structure}

Wendelstein 7-X is structured on the basis of the KKS-system (Kraftwerk-

Kennzeichensystem = Identification System for Power Plants), which provides a unique alpha-numerical identification of all parts of the device using a four level hierarchy (plant function - aggregate - equipment). Components of W7-X are allocated on the "function"levels which again have their own internal hierarchy, e.g. torus (A) - magnet system (AA) non-planar coils (AAB) - non-planar coil type 1 (AAB01) - non-planar coil type 1 in module 5 (AAB51). Generally, the system specification is concerned with the higher "function"levels (e.g. A, AA, AAB), whereas interface coordination starts at the lowest but still general level (e.g. AAB). The structure is administrated by the department Documentation. Changes and amendments are agreed between the departments Documentation, Design Office and Configuration Management.

\subsection{System specification}

The original system specification of W7-X specifies

- the scientific goals of W7-X

- the performance attributes of the project

- the technical components required to reach these goals

- the performance attributes of the components

- general boundary conditions and rules, technical and legal requirements

- rules for assembly and maintenance

- the foreseen modes of device operation.

With the introduction of a PLM-system into the project it appeared to be no longer adequate to update the original system specification as a monolithic document, but instead to follow a distributed approach which makes most efficient use of PLM supported features such as release control, messaging service, use of keywords, linking of documents and others.

Therefore a PLM-based navigation tool is currently being set up, which - starting from master documents at the component level - guides the user to the information that completely 
specifies the current status of the component including its interfaces to other components and additional background information:

- the position of the component in the project structure

- the position of the component in the CAD assembly

- the original specifications and data sheets as the initial configuration baseline

- the history of design changes

- the history of variances arising from quality deviations

- the interface descriptions

- additional references of relevance, e.g. calculations in support of design, safety analyses etc.

This concept resembles a classical loose-leaf collection, in German "Ringbuch", and the master document which is the central reference for a component is therefore termed "Ringbuch". In a similar way additional Ringbuch documents are being generated on a topical level, which specify more general aspects as for example the W7-X coordinate system, magnetic field maps within the cryostat and the torus hall for the various magnetic configurations, load cycles of W7-X, etc.. Electronic links between master document and referenced documents ensure that revisions are automatically visible for the user.

\subsection{Interface coordination}

Interfaces transfer both the dedicated functional interactions and the unintended parasitic interactions between two components. Unambiguous specification of the functional interaction is prerequisite to assure the intended operability of an interface. Parasitic interactions can inevitably arise from the co-existence of components and may require counter measures to be specified - for example shielding, insulation, sufficient clearance - in order to prevent their unfavourable impact or reduce it to a tolerable level. They must be considered from the beginning in order to avoid interface conflicts. Thorough interface control is prerequisite to ensure the functionality of interfaces and to identify interface conflicts in advance during all phases of the project, i.e. design, construction and operation.

The tasks of interface control for W7-X are

- identification of all interfaces via the interface matrix of components 
- specification of the interfaces between each pair of components in a standardized interface description document

- verification of interface descriptions

- identification of open interface issues and interface conflicts

- tracking the status of interface descriptions, interface conflicts and open issues

- assessment of change impact across the interfaces

- information of the relevant persons on status and changes of interfaces

An interface description document contains five sections: (1) the definition of the pair of interfacing components, (2) the status table showing the current status of the interface, (3) the interface document table, which lists all additional documents required to completely specify the interface, (4) the interface description, (5) the list of open interface issues with tasks, responsible persons and due dates to resolve this issues. According to the specific interactions of components interfaces are classified into 15 categories which are described as separate items in the status table and the interface description section: geometric, mechanical, electrotechnical, magnetic, thermal, control and data aquisition, cryogenic, vacuum-technical interfaces, and others. Collision control of geometrical interfaces is subjected to dedicated investigations performed by the department Configuration Space Control [2] and is not included in interface coordination. The interface description document defines both the technical interface parameters as well as the organizational responsibilities at the interface. The document is generated and approved by the responsible officers of the components involved and by CM. CM finally releases the document and notifies the relevant persons.

The interface documents are electronically administrated via the PLM system. Interface status information and open issues are stored in a data base to support interface control. In addition, interface related tasks are transferred from the data base to the work breakdown structure of the responsible officers. Whenever a task is completed the interface description document is accordingly revised. The revision is released when a significant level of completion is reached. Currently the W7-X interface matrix is built up by about 60 components. About 160 pairs of them have identified interfaces which are followed up by the interface coordination process. Interfaces on the aggregate level which are completely embedded within the higher level component are not considered, as long as they do not affect the boundary of the main component. These internal interfaces are in the responsibility of the responsible officer (RO). 


\section{Change management}

The configuration of a complex project like W7-X steadily evolves during its life cycle by intentional design changes and amendments, and by residual variances to the initial design. The latter may arise during fabrication or assembly from non-conformances to specifications but cannot not be completely removed by repair measures. Control of the respective change and non-conformance processes is guided by $\mathrm{CM}$ which includes that $\mathrm{CM}$

- enables change and non-conformance decisions to be based on knowledge of complete change and non-conformance impact

- limits changes to those which are necessary or offer significant benefit

- facilitate evaluation of cost, savings \& trade-offs

- ensures the scientific interests of the project are considered

- preserves configuration control at the interfaces

- provides orderly communication of change information

- maintains and controls a current configuration baseline

- maintains consistency between product and documentation

- ensures documentation of all variances

Changes are typically requested in order to reduce cost and/or time, to improve or ensure performance attributes of a component, to remove interface conflicts, to ease or facilitate manufacturing processes or to ensure mountability and maintainability. An essential amount of change requests arises from the considerable risk of geometrical collisions of components, e.g. coils and thermal insulation, within the limited space of the cryostat. This risk is thoroughly analysed by the Configuration Space Control (CC) department using as-built models of the most critical components (superconducting coils, support structure) and design models otherwise (thermal insulation, superconducting busbar, cryo pipes). Imposed to the models are the deformations and movements of the magnet system as predicted for the various operational regimes of W7-X by the global structural model [3, 4]. Whenever a collision is detected a change request is initiated by $\mathrm{CC}$ which specifies how to change the geometry of one (or both) of the colliding components in order to remove the risk of collision. 
In the current phase of the W7-X project some components are still under procurement or even under design while the assembly of the device is steadily proceeding along a tight schedule. In order to prevent delay of device assembly due to change and non-conformance processes an organizational structure has been established with escalating decision paths which lead from the occurrence of a change request or detection of a non-conformance to the implementation of the corresponding measures (see Fig.1). These paths are adapted to the criticality of the individual problem. Initially, the criticality is assessed by the project team of the affected component ( $\mathrm{RO}$ of component, $\mathrm{RO}$ of assembly step, assigned member of Quality Management). If the problem turns out to be routine, i.e. a qualified solution already exists and does not affect cost and time, it can be implemented immediately including a proper documentation. If not, CM is involved and the time criticality is assessed in the next step. If the problem is not time critical, the RO leads the subsequent process which either starts with a formal change note $(\mathrm{CN})$ or with a non-conformance report (NCR). These documents apply to implement the requested change or to repair or to tolerate the non-conformance. This path is the standard case and change notes and NCR are assessed and approved by the members of the W7-X board and by experts. If the problem is considered time critical, CM leads and accelerates the further process. This includes provision of the complete information required for the problem to be assessed by the Configuration Control Board (CCB), which is called adhoc and chaired by the technical director. It decides on the measures to be taken. These are finally documented in the $\mathrm{CN}$ or the NCR (which in very urgent cases are to be generated after the decision). In all cases the RO of the respective component or the assembly step is responsible to implement the decision.

CM monitors all CN- and NCR-processes, controls that schedules are kept, pushes slowly proceeding processes by direct interaction and continuously updates the information on the process status. For this purpose the basic process data are registered in a data base, which allows control of individual processes and production of up-to-date lists of component related $\mathrm{CN}$ and NCR that are linked to the "Ringbuch". A process is finally closed by CM when all documents which are affected by the decision have been updated: CAD models, drawings, quality assurance and assembly plans, interface descriptions and others. CM ensures that all persons involved are informed about release of a measure and closure of the process. After closure CM and the department Device Safety assess possible impacts of changes or nonconformances on the operation of $\mathrm{W} 7-\mathrm{X}$ and attribute a risk level. The risk evaluation results 
are stored in the data base and will later on support error analysis and trouble shooting in case of events of fault during operation.

\section{Summary and conclusion}

Within 14 months after implementation of CM for W7-X an integrated concept for system documentation and change management has been established. Maximum use of existing tools and processes has been made wherever possible. Links between all CM-relevant documents in the product lifecycle management system support the consistency of the system documentation including the impact of design changes and variances. The PLM-system is simultaneously used as a documentation data base, as a navigation platform to the specification of W7-X and its components and for notification on new releases of CMrelevant document, for example specifications, interface descriptions, change notes and nonconformance reports. Permanent control of the various CM processes is supported by a specific process data base. As an essential result decision processes have generally been accelerated and decisions, their rationales and impacts are efficiently communicated within the project. All problems that have been time critical with respect to the assembly schedule could be decided by the configuration control board without delay being introduced by the decision process itself.

\section{References}

[1] L. Wegener et al., Status of Wendelstein 7-X Construction, this conference

[2] Quality management systems - Guidelines for configuration management, ISO 10007:2003

[3] C. Baylard et al., Configuration Space Control for W7-X, this conference

[4] V. Bykov et al., Structural analysis of W7-X: Main results and critical issues, Fusion Engineering and Design 82 (2007) 1538-1548 
Fig. 1 Decision paths for change and non-conformance processes

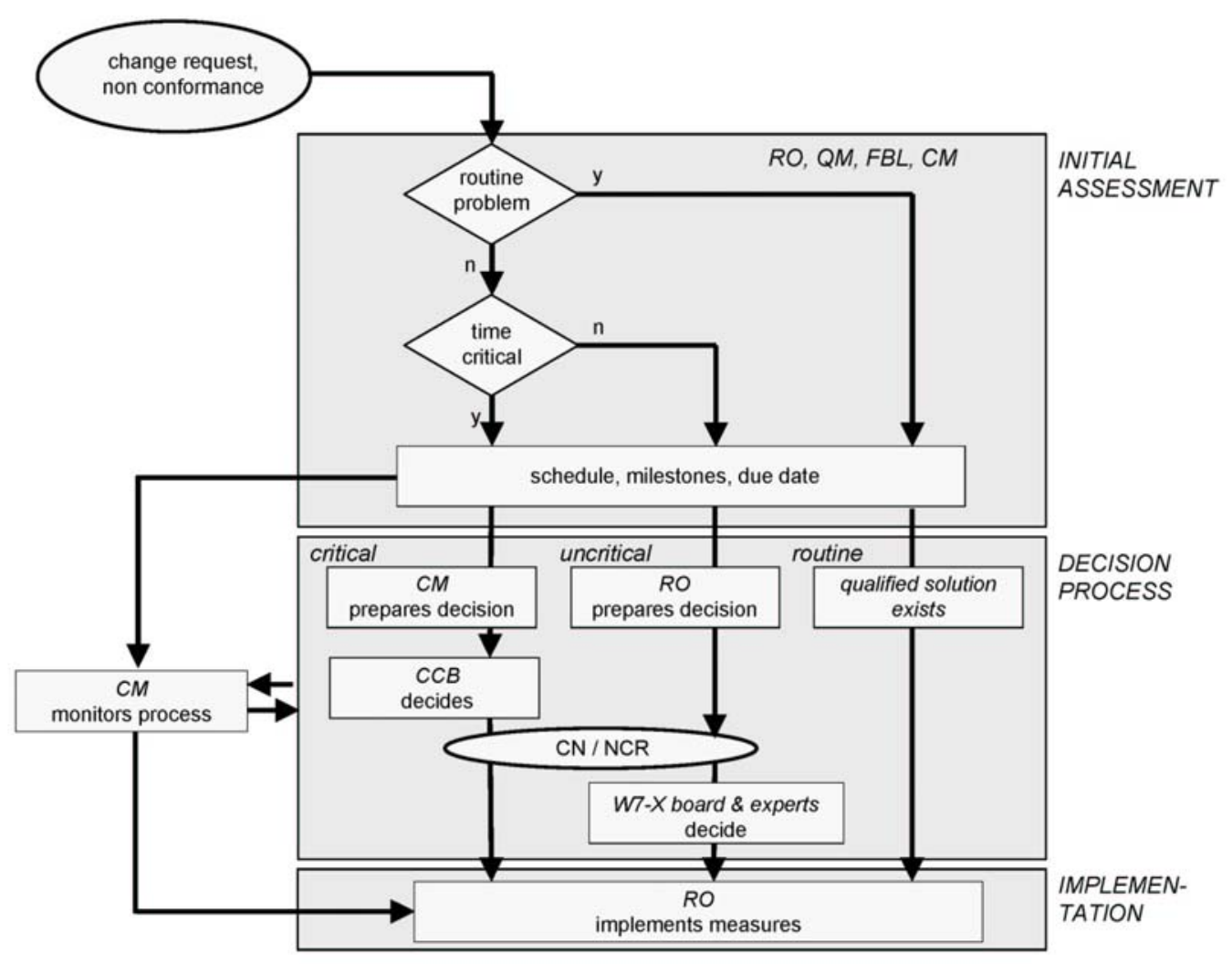

